\title{
1 A spinner magnetometer for large Apollo lunar samples
}

M. Uehara1, a), J. Gattacceca ${ }^{1}$, Y. Quesnel ${ }^{1}$, C. Lepaulard ${ }^{1}$, E. A. Lima ${ }^{2}$, M. Manfredi ${ }^{3}$, and P. Rochette ${ }^{1}$

${ }^{1}$ CEREGE, CNRS, Aix Marseille Univ, IRD, Coll France, CEREGE, 13545 Aix-en-Provence, France

${ }^{2}$ Department of Earth, Atmospheric and Planetary Sciences, Massachusetts Institute of Technology, Cambridge, MA 02139, USA

${ }^{3}$ CGI, 92097 La Défense, France.

auehara@cerege.fr

10 Abstract

We developed a spinner magnetometer to measure the natural remanent magnetization of large Apollo lunar rocks in the storage vault of the Lunar Sample Laboratory Facility (LSLF) of NASA.

13 The magnetometer mainly consists of a commercially available three axial fluxgate sensor and a

14 hand-rotating sample table with an optical encoder recording the rotation angles. The distance 15 between the sample and the sensor is adjustable according to the sample size and magnetization 16 intensity. The sensor and the sample are placed in a two-layer mu-metal shield to measure the sample

17 natural remanent magnetization. The magnetic signals are acquired together with the rotation angle 18 to obtain stacking of the measured signals over multiple revolutions. The developed magnetometer 19 has a sensitivity of $5 \times 10^{-7} \mathrm{Am}^{2}$ at the standard sensor-to-sample distance of $15 \mathrm{~cm}$. This sensitivity 20 is sufficient to measure the natural remanent magnetization of almost all the lunar basalt and breccia 21 samples with mass above $10 \mathrm{~g}$ in the LSLF vault.

Key words: magnetometer, remanent magnetization, Apollo samples, geophysics

\section{RATIONALE}

The Moon has no global magnetic field today. However spacecraft observations have shown that large portions of the crust are magnetized (e.g., Purucker and Nicholas ${ }^{1}$ and Tsunakawa et al. ${ }^{2}$ ). Paleomagnetic studies of samples

27 returned by the Apollo program have also shown that some of these rocks carry a significant remanent magnetization that

28 was acquired on the Moon ${ }^{3}$. It is now rather firmly established that the Moon once had a global magnetic field generated

29 by a dynamo mechanism in a molten metallic core ${ }^{4}$. However, crucial questions remain to be answered such as the

30 intensity of the lunar paleofield, its geometry, and the exact timing of the dynamo onset and turn-off. Answering these

31 questions would ultimately shed light on the interior structure of the Moon, on the processes that allowed dynamo

32 generation, and would provide the dynamo theory with a robust test case. 
A fairly large number of samples (74) were studied in the 1970's, soon after their return from the Moon (Fuller and 34 Cisowski $^{3}$ for a review). A new series of more refined paleomagnetic and thermochronology studies have been 35 performed in the 2010's ${ }^{5-9}$. All together, about 71 different Apollo rocks (for a total of 90 samples) have been studied for 36 paleomagnetism. This represents only $5 \%$ of the 1402 individual returned during the Apollo program. All these studies 37 (with the exception of Cournède et al. ${ }^{6}$ ) were performed on small chips (usually $<1 \mathrm{~g}$ ) allocated for detailed laboratory 38 work that generally include sub-sampling and study of even smaller fragments using high-sensitivity Superconducting 39 Quantum Interference Device (SQUID) magnetometers ${ }^{10-12}$. Therefore, these paleomagnetic studies imply destructive 40 and time-consuming sub-sampling of the original Apollo rocks by curators and processors at NASA. Consequently, an 41 exhaustive paleomagnetic study of the Apollo collection appears out of reach using standard procedure.

\section{Specificities and interest of the proposed measurements}

With the aim of making an exhaustive magnetic survey of the Apollo rocks, we adopted the following strategy: perform simple magnetic measurements (Natural Remanent Magnetization, NRM) of the whole, unprocessed sample directly in the Lunar Sample Laboratory Facility (LSLF) storage facility, without any subsampling or demagnetization, thus reducing sample preparation and handling to a minimum that is acceptable for curators. Measuring large whole samples has other advantages in addition to its non-destructive quality. First, lunar rocks can be heterogeneously magnetized, especially the breccias that make up a large fraction of the Apollo collection. Indeed, different parts of a lunar breccia (matrix, clasts of various lithiologies, melt) can have strongly contrasted magnetic properties, and also different paleomagnetic direction if the magnetization of the clasts has survived the assembly of the breccia. Second, the study of small sub-samples increases the apparent effect of possible remagnetization during sample return or processing. Some samples have been shown to have been partially and locally remagnetized by exposure to fields up to several $\mathrm{mT}$ during the return flight from the Moon (e.g., Pearce et al. ${ }^{13}$ ). Others have been locally heated during cutting with band saw ${ }^{14}$. Studying whole large samples will minimize the bulk effect of these magnetic contaminations, given that they can dominate the signal when studying small samples that may come from the area that has been heated of exposed to a strong field. The aim of our study is chiefly to perform an exhaustive survey of the NRM of Apollo rocks to identify the key samples that can then be studied in details in the laboratory using standard paleomagnetic techniques. Therefore, we needed to develop a magnetometer that could measure the magnetic moment of whole unprocessed Apollo samples directly in their storage facility, while complying with NASA curatorial constraints. 
size) filled with pure nitrogen gas to avoid oxidation and contamination. Sample mass ranges from $<1 \mathrm{~g}$ to about $5 \mathrm{~kg}$. In

64 this study, we focused on samples above $50 \mathrm{~g}$, corresponding to about $15 \mathrm{~cm}^{3}$. The Apollo collection contains about 200

65 of such samples. Among them, only about 40 have been studied for paleomagnetism so far, indicating that an exhaustive

66 survey will likely bring new valuable information.

\section{Instrumental constraints}

Although modern commercial SQUID magnetometers are perfectly adapted for detailed paleomagnetic studies of lunar rocks, they can typically only accommodate samples up to about $10 \mathrm{~cm}^{3}$ (about $30 \mathrm{~g}$ ) and are not portable, making them unsuitable for the proposed measurements. We need a magnetometer optimized for fast and efficient measurements of whole lunar rocks in the vault. The instrumental precision and accuracy are not the main constraints, since this instrument is mostly designed for the purpose of triage of samples for further more refined analyses in the laboratory.

There are five technical challenges for the development of a magnetometer able to measure the NRM of unprocessed Apollo rocks in situ in their storage vault. The first is the limitations imposed by the curatorial constraints. As mentioned, samples must remain in their original packaging to avoid any chemical contaminations and time-consuming repackaging by NASA processors. Moreover, many mechanical components and chemical compounds (gear, cam, slider, electric motor, metal ball bearings, oils, etc.) cannot be used in the vault to avoid chemical contamination. This limitation requires that the magnetometer must use very simple mechanisms. The second constraint is the wide range of the expected magnetic moments to be measured due to the variety of sample size and nature. Depending on the lithology, the NRM is expected to vary from weak (norite, anorthosite, $\sim 10^{-7} \mathrm{Am}^{2} / \mathrm{kg}$ ) to relatively strong (basalt, $\sim 10^{-5} \mathrm{Am}^{2} / \mathrm{kg}$; impact melt breccias, $\left.\sim 10^{-4} \mathrm{Am}^{2} / \mathrm{kg}\right)^{6}$. Because we focus mostly on samples that range from $40 \mathrm{~g}$ to $4 \mathrm{~kg}$ in mass, the variation between the weakest and the strongest samples can be in the order of $10^{4}$, requiring a wide dynamic range. The third constraint is sensitivity, which must be good enough to allow measurement of the NRM of most Apollo rocks with mass above $50 \mathrm{~g}$. The fourth constraint is portability. To be allowed access to the lunar vault, the magnetometer should be dismountable, compact, and easy to reassemble in the vault. The fifth constraint is processing speed because hundreds of samples must be measured. Working in the vault requires the continuous presence of a NASA lunar curator and/or processor, and represents a heavy load in terms of personnel use. Measuring 100 samples in a week, including initial setup and final disassembly of the magnetometer, implies that the measurement time (including sample handling) has to be 10 minutes per sample at most. 
magnetic field sensor, a sample on a rotating stage, magnetic shields enclosing the sensor and the sample, and an encoder

94 detecting the rotation angle. The sample, ideally carrying a magnetization equivalent to a single dipole, generates

95 sinusoidal signals for the radial and the tangential components of the field at the sensor position as the rock is rotated

96 about the vertical axis. By changing the sample's orientation at least two times, we can estimate the three components of

97 magnetic moment. We can adjust the sensor-to-sample distance to measure samples of various sizes and achieve large

98 dynamic range. Moreover, we can improve its signal-to-noise ratio $(\mathrm{S} / \mathrm{N})$ by stacking the data during multiple revolutions

9917,18 . In this paper, we describe a portable spinner magnetometer developed specifically to measure the magnetic

100 moments of large unprocessed Apollo samples in the LSLF vault. Furthermore, we present pilot data processing using

101 the result of the actual measurement of 133 Apollo samples during a first round of measurements in the LSLF, in

102 addition to performance tests in our laboratory.

\section{DESCRIPTION OF THE MAGNETOMETER}

Figure 1 shows schematic illustrations of the spinner magnetometer for the large Apollo samples. A commercial three-axis fluxgate magnetic field sensor (Mag-03MS100, Bartington Instruments Ltd.) and a rotating sample stage are enclosed in a two-layer mu-metal magnetic shield $(550 \mathrm{~mm}$ in diameter and $500 \mathrm{~mm}$ in height). The interior of the magnetometer can be accessed by opening the top lids of the mu-metal shields. To minimize stray fields, all of the holes penetrating the both inner and outer shield are arranged not to be co-axially positioned, except for the $11 \mathrm{~mm}$ bore for the spindle and the $4 \mathrm{~mm}$ hole for the feedthroughs. The residual magnetic field, which is mainly the stray field resulting

112 the magnetic field, as evaluated during 10 successive opening and closing operations of the mu-metal shield. The 113 samples are enclosed in a cube made of transparent acrylic resin (PMMA) plates welded by solvents. Cubes with 114 different dimensions $(5,7,10,12,15,17$, and $20 \mathrm{~cm}$ sides) were used to best fit various sample sizes and shapes.

115 Samples are kept tight in the cubes using Teflon films and/or PMMA rings. The cubes have center marks on the surfaces 116 that help locating the sample at the center. For samples with anisometric shapes, we recorded the shape and position in 117 the cube for the later more refined analyses. As shown in Fig. 1b, the center of the cube is at the intersection of the 118 spindle of the stage and the horizontal centerline of the fluxgate sensor (hereafter call "stage center"). When using the 119 smaller cubes, acrylic resin spacers are used to keep the cube center at the stage center. The distance between the stage 120 center and the sensor $(d)$ is adjustable according to the magnetic moment intensity and the size of the sample. The sensor 121 holder can be fixed by an aluminum pin on the guide rail that has bores at $d=15,16,17.5,20,22.5,25,27.5$, and $30 \mathrm{~cm}$. 122 The sensor can be moved as close as $d=5 \mathrm{~cm}$ from the sample center by using a PMMA extension plate. The sample 
123 stage is revolved manually using an aluminum handle directly connected to the spindle via an aluminum coupling 124 mechanism. The target turning speed is about 1 revolution per 10 seconds $(0.1 \mathrm{~Hz})$, which is slower than other magnetic125 sensor-equipped spinner magnetometers $(5 \text { to } 7 \mathrm{~Hz})^{19}{ }^{20}$. To avoid chemical contamination, lubricant-free Teflon 126 bearings were used. All the other metallic parts are made of aluminum, except for the mu-metal shield, which is never in 127 contact with the PMMA cubes containing the samples.

128 Figure 2 shows the schematic operation diagram of the magnetometer. The fluxgate sensor has output noise spectral 129 densities of about $9 \mathrm{pT}_{\mathrm{rms}} \cdot \mathrm{Hz}^{-0.5}$ at $1 \mathrm{~Hz}$ for three components and the orthogonality errors are $<0.1^{\circ}$, according to the 130 manufacturer specifications. The rotation angle of the spindle is measured by an optical encoder connected to a digital 131 input/output interface (NI 9403, National Instruments Corp.). The resolution of the encoder is 512 pulses-per-revolution 132 and the maximum position error is $0.167^{\circ}$. The digital back-end of the encoder can handle rotation speed up to several 133 thousand rotations per minutes. Moreover, the index signal force to reset the decoder's counter, preventing propagation 134 of counting error. A four-channel 24-bit A/D converter unit with $\pm 10 \mathrm{~V}$ measurement range (ADC; NI 9239, National 135 Instruments Corp.) samples all three channels (X-, Y-, and Z-axis) simultaneously after amplified (Gain = 1000) and 136 conditioned by a signal conditioning unit (SCU; SCU-3, Bartington Instruments Ltd.). The analog and digital front end 137 units are mounted on the USB chassis (NI cDAQ-9174, National Instruments Corp.) that can realize a synchronous 138 operation of the mounted units.

139 Figure 2 also shows a block diagram of the data acquisition software. The entire acquisition process is controlled by 140 a 64-bit LabVIEW (National Instruments Corp.) program running on a laptop PC. Since the revolution speed of the 141 sample is variable, the synchronization between the encoder position and the sensor signals is important to measure the 142 magnetic field distribution around the sample accurately. The program has two parallel threads working as a real-time 143 routine. The first thread controls the sampling and simple low-pass filtering. To avoid the problem of aliasing, the ADC 144 oversamples the signals at 50k samples per second (sps) that is 500 times faster than the cut-off frequency of the SCU's 145 second ordered low-pass filter $\left(f_{c}=100 \mathrm{~Hz}\right)$. The digitized data stream is stored in a buffer and re-sampled at 50 sps by 146 averaging of the buffered 1000 samples, which plays as a digital low pass filter that removes signals above $50 \mathrm{~Hz}$. This 147 data stream is double buffered not to drop any data during unexpected heavy forward processes. The second thread 148 records the position of the optical encoder through communications with the decoder that returns the position of the 149 optical encoder's index mark with a 512 pulse-per-revolution resolution. The standard direction of rotation is defined as 150 clockwise (CW). The gating of the sampling and the acquisition of the encoder position is triggered by the shared $50 \mathrm{~Hz}$ 151 software trigger, realizing a synchronous measurement of the magnetic field and the sample position. The resolution of 152 the optical encoder (512 positions/revolution) and the data acquisition frequency (50 Hz) is optimized for the target 

rotation speed that will not be affected by the SCU's low-pass filter is $1000 \%$, which is five times faster than the

155 instantaneous rotation speed in the actual measurements (see Appendix A). The data are recorded along with timestamps 156 of $1 \mathrm{~ms}$ precision for the purpose of the post-acquisition filtering processes. Finally, the dataset is saved on the hard disk.

\section{THEORY OF OPERATION}

This magnetometer measures the magnetic fields around the rotating sample. For simplicity, we consider a dipole moment vector $\boldsymbol{m}=\left(m_{x}, m_{y}, m_{z}\right)$ at the center of the sample cube. We define the sample coordinates as following. We defined the north, east, and down surface of the sample cube that respectively correspond to x-, y-, and z-axis directions

162 (Fig.2). Using declination $D$ and inclination $I$, this vector can be written as $\boldsymbol{m}=(m \cos D \cos I, m \sin D \cos I, m \sin I)$, where $m=|\boldsymbol{m}|$. We can observe sinusoids that are functions of the rotation angle $\theta$ due to the rotation of $\boldsymbol{m}$. The Y-, X-, and Z-

164 axis of the fluxgate sensor measure the radial, tangential, and vertically downward components of the field, respectively (Fig. 2). The observed magnetic field vector $\boldsymbol{B}(\theta)$ is given by

$$
\left\{\begin{array}{c}
B_{X}(\theta)=\frac{m \cos I}{4 \pi} \times \frac{-\sin (D+\theta)}{d^{3}} \\
B_{Y}(\theta)=\frac{2 m \cos I}{4 \pi} \times \frac{\cos (D+\theta)}{d^{3}} \\
B_{Z}(\theta)=\frac{m \sin I}{4 \pi} \times \frac{1}{d^{3}}
\end{array}\right.
$$

Note that the $\mathrm{CW}$ rotation of the stage makes the scanning direction of the sample counter clockwise (CCW), resulting in

168 a negative sign of the term $\sin (D+\theta)$. We defined the origin of the rotation $\theta$ when the sample north points toward the sensor. We can calibrate the stage north by measuring a point source placed on the north notch of the stage (Fig. 2); the position where $\left|B_{X}+B_{Y}\right|$ becomes maximum corresponds to the north $(\theta=0)$. It is important to note that the waveform of the $B_{Z}$ component is constant and $B_{X}$ and $B_{Y}$ components are sinusoidal. Unfortunately, our magnetometer cannot

172 measure $B_{z}$ directly due to the DC offsets. Thus, we change the sample position in three different rotation axis; around z-

173 axis (position 1), y-axis (position 2), and x-axis (position 3). The acrylic cubic sample holders have been checked for 174 precise orthogonality to ensure the accuracy of these orthogonal rotations. This operation enables to measure all three 175 components of the moment $\boldsymbol{m}$ as sinusoidal signals and solves the problem of the DC offsets. For this reason, the DC 176 component is not considered in the post-processing, and the chart always starts from $0 \mathrm{nT}$ at the beginning of the 177 measurement.

178 The encoder angle is sampled at a fixed frequency $(50 \mathrm{~Hz})$ that is asynchronous to the optical encoder's movement 179 (Fig. 2). This asynchronous sampling makes a quantization error between the actual direction $\theta$ and the apparent encoder 
angle $\theta_{\text {enc }}(n)=n \times 2 \pi / N$, where $n$ is the encoder count $(n=0,1, \ldots \mathrm{N}-1)$ and $N$ is the number of the pulses per revolution, yielding the resolution of the encoder $\Delta \theta=2 \pi / N(\mathrm{rad})$. This quantization error $\theta_{\text {err }}=\theta-\theta_{\text {enc }}(n)$ is randomly distributed in

182 the range $0 \leq \theta_{\text {err }}<\Delta \theta$, which makes a signal error given by $\operatorname{err}\left(\theta, \theta_{\text {err }}\right)=\boldsymbol{B}\left(\theta+\theta_{\text {err }}\right)-\boldsymbol{B}(\theta)$. This is akin to quantization noise. The worst-case signal error is approximately given by substituting $\Delta \theta$ for $\theta_{\text {err }}$. For an encoder with a good resolution (e.g. $N>50$ ), this worst-case signal error is

$$
\operatorname{err}(\theta, \Delta \theta)=\Delta \theta \times a\left(\theta_{\text {enc }}(n)\right)(2),
$$

where $a\left(\theta_{\text {enc }}(n)\right)$ is the slope of the signal at the $n$-th encoder position. This worst-case error can reach $2 \pi A / N$ at the

187 maximum when we measure a dipole magnetic field with an amplitude $A$, given by $B(\theta)=A \sin (\theta)$ (see Appendix A).

188 The first remedy to reduce this error is simply increasing the encoder's resolution $N$. The second is simultaneous 189 acquisition of the optical encoder and the $\mathrm{ADC}$ to keep the same $\Delta \theta$ value during the measurement, because this error is a 190 sort of a phase error. The third is calculating an average during the passage between two positions, improving the worst191 case error in half ( $\pi A / N$, see APPENDIX A); this technique is eventually realized by the oversampling method (Fig. 2).

192 To conclude, the current system with $N=512$ has a worst-case error of $\pi / 512=0.61 \%$ of the amplitude, which is $6 \mathrm{pT}$ 193 for a typical $A=1 \mathrm{nT}$ signal. This is below the output noise density of the fluxgate sensor and far below the ambient noise (several tens of pT), indicating that it is negligible in our system.

One of the advantage of the spinner magnetometer is that the signal-to-noise ratio $(\mathrm{S} / \mathrm{N})$ can be improved by post processing. This spinner magnetometer conducts a box-car integration (stacking) of the magnetic field signals whose reference signal is the encoder output. By filtering and stacking of the data over multiple revolutions, we can decrease the noise, which is not synchronized with the rotation of the sample, unlike the periodic signal resulting from the magnetization of the sample ${ }^{17,18}$. We developed Python scripts using Scipy library (www.scipy.org) that conducts three steps of post processing. Figure 3 shows an example of the post processing using the dataset of the Apollo 12 sample (No.

201 12018.15) measured in the LSLF vault. The first step is removing low-frequency noise components whose frequencies 202 are lower than that of the revolution (drifting and baseline jumping) due to temperature drifts and disturbance of the 203 ambient field, which may be dominant in the untreated signal (left-side chart of Fig.3a). To remove this low-frequency 204 noise, we subtract the baseline from the signal. The baseline is estimated by the application of a Savitzky-Golay filter with $1^{\text {st }}$ order polynomial fitting and 32 points window. The baseline for the first and last 32 points, where we cannot apply this filter, is estimated by a linear approximation. The right-side chart of Fig.3a shows the signals after subtracting the baseline, indicating the successful removing of the targeted noises. The second step is stacking (Fig. $3 \mathrm{~b}$ ). In the 208 stacked result, we can roughly identify the sinusoidal curve buried in high-frequency noises. The third step is the lowpass filtering by a fast Fourier transformation (FFT). Since we try to explain the magnetic field by a single dipole source 
211 than $100^{\circ}$ wavelength, which can be originated fine-scaled magnetic structure, high-frequency noises, or non-dipole

212 component ${ }^{20}$, are removed by FFT filter after smoothing by a weak Savitzky-Golay filter with $1^{\text {st }}$ order polynomial

213 fitting and 11 points window. The solid line in Fig.3b is the waveform after this FFT filtering. Since the stacked

214 waveform is averaged over multiple periods, it is enough continuous at both ends to carry out FFT. This stacked and

215 filtered waveforms are used for the inversion to predict the dipole source parameters. This last step consists in a standard

216 least-square inverse approach to find the best-fitting set of the 3 unknown parameters: dipole moment intensity,

217 inclination and declination. The dipole is assumed to be centered. Indeed, our results show that $90 \%$ of the samples show

218 magnetic field measurements 'coherent' (i.e. less than $20 \%$ of error between predictions and observations) with a dipolar

219 source located at the center of the sample, though the rest $10 \%$ of the samples contain quadrupole or higher harmonics

220 probably due to the very anisotropic shape or heterogeneous composition like lunar impact breccia. Off centered dipole

221 may also be the source of non-dipole character ${ }^{20}$.

222 During the measurements, the LabView program displays the raw data after stacking with error bars (+/- standard 223 deviation) as a plot versus rotation angle $\theta$. Note that we visualize a result of stacking without filtering to reduce the CPU

224 load and keep the real-time routine. The program also shows the estimated sinusoidal curve and the noise level, which are

225 calculated by FFT results of the observed signal. The noise will reduce with stacking inversely with the square root of the

226 number of revolutions. The user can stop the rotations of the sample when the quality value cannot improve any further

227 by adding revolutions. The nominal revolution time is about 8 turns (1.5 minutes) and thus the noise is reduced by $65 \%$

$228\left(=8^{-0.5}\right)$ theoretically ${ }^{17}$. The user also can check the skewness of the sinusoidal curve, which can originate from the shape

229 effect or inhomogeneity of NRM, and increase the sensor-to-sample distance to reduce those multipole components.

\section{VI. PERFORMANCE OF THE MAGNETOMETER}

232 Table 1 and Figure 4a show the result of a demagnetization experiment at the CEREGE laboratory (Aix-en-Provence, 233 France) using the large sample spinner magnetometer and a commercially available SQUID magnetometer with an in234 line alternating field (AF) demagnetizer (2G Enterprise, model 760R). A small terrestrial basalt fragment (0.98 g), which 235 can be considered as a quasi-dipole source, is enclosed in a 1 inch cubic plastic capsule. The sample is measured with the 236 spinner magnetometer using a three-position scheme (i.e., rotation around $\mathrm{x}-, \mathrm{y}-$, and z-axis), and then, it is also measured 237 with the SQUID magnetometer and demagnetized by the AF. We continue this sequence up to $80 \mathrm{mT} \mathrm{AF}$ 238 demagnetization field to check for the effect of variable magnetic moment intensity. In view of the high precision of the 239 SQUID magnetometer $\left(2 \times 10^{-11} \mathrm{Am}^{2}\right)^{21}$, and its cross calibration with other magnetometers in our laboratory (including 
241 close enough to the actual magnetic moment intensity for the intensity range in this study $\left(10^{-6} \mathrm{Am}^{2}\right)$. The predicted

242 intensities of the dipole moment using our spinner magnetometer are in close agreement with the actual dipole moments,

243 though there is some overestimation between $0.8 \%$ and $7.0 \%$ (Fig. 4a). Since the amount of the overestimation is not a

244 function of the intensity of the magnetic moment, it seems that this error is not due to the noise but other factors such as

245 positioning error when we replace the samples at each step. It is notable that the error in the direction is also small (from

$2463^{\circ}$ to $10^{\circ}$, Table 1). The cubic shape of the sample capsule can constrain the tilt (inclination) of the sample but let freely

247 rotate horizontally (declination) during the repeated placing of the sample. This may explain the larger error in 248 declination (from $-1^{\circ}$ to $+12^{\circ}$ ) than in inclination (from $+2^{\circ}$ to $+3^{\circ}$ ).

249 We estimated the repeatability error of this instrument by five repeated measurements of this basalt sample. Due to 250 our operational schedule, we conducted this experiment within a magnetically shielded room of the CEREGE laboratory 251 but without the mumetal shield of the instrument. This configuration increases the background field and noise by a factor 252 of ten. The sample was saturated in a $1 \mathrm{~T}$ magnetic field generated by a pulse magnetizer (model MMPM-9, Magnetic 253 Measurements Ltd.). The standard deviation for the five measurements is $3 \%$ of the average magnetization of the sample 254 (Table 2). The semi-angle of aperture of the $95 \%$ confidence cone $\left(\alpha_{95}\right)^{22}$ is $1.7^{\circ}$, which gives one angular standard 255 deviation $( \pm 1 \sigma)$ of $2.2^{\circ}$. These results indicate a satisfactory repeatability of this instrument. We also conducted a series 256 of measurements at four different sensor-to-sample distances. The result shows similar variability as for the repeatability 257 test (Table 3). This indicates that the error due to the different distance is within of the error due to the repeatability. 258 Overall, the intensity and directions provided by the instrument are precise within $3 \%$ and $2^{\circ}$, respectively, and likely 259 better than that when using the mutmetal shielding.

260 Figure $4 \mathrm{~b}$ shows the example of the severe $\mathrm{S} / \mathrm{N}$ condition of sample demagnetized by $80 \mathrm{mT}$ AF. The peak-to-peak 261 noise at CEREGE experiment is $250 \mathrm{pT}_{\mathrm{p}-\mathrm{p}}$ and that at NASA (Fig. $3 \mathrm{~b}$ ) is $203 \mathrm{pT} \mathrm{T}_{\mathrm{p} p}$ that is $20 \%$ weaker than in CEREGE. 262 Carefully observing the result at LSLF, there is no spike noise such the one visible in the result at CEREGE. This low 263 noise environment at the LSLF vault is due to the fact that the vault itself is equivalent to a closed stainless-steel capsule 264 which acts as a good electromagnetic shield. As demonstrated by a previous study, it is hard to recover the signal buried 265 in a strong noise. Using these background noise data, we try to estimate the worst $\mathrm{S} / \mathrm{N}$ for which we can still recover the 266 signal. The $\mathrm{S} / \mathrm{N}$ is defined as (root mean square amplitude of signal) / (standard deviation of noise). We can assume that 267 the forward model using equation (1) and the estimates by the SQUID measurement can be the actual signal without 268 noise. The noise can be estimated by the difference between this forward model and the observed signal after stacking. 269 Because the $\mathrm{S} / \mathrm{N}$ for $B_{X}$ is simply half that of $B_{Y}$ (eq. 1), we consider only $B_{Y}$ now. The amplitude of $B_{Y}$ given by the 
SQUID measurement is $16.7 \mathrm{pT}_{\mathrm{rms}}\left(47.7 \mathrm{pT}_{\mathrm{p}-\mathrm{p}}\right)$ and the standard deviation of the noise is $33.5 \mathrm{pT}_{\mathrm{rms}}$, giving $\mathrm{S} / \mathrm{N}=0.50$.

271 We can also calculate the $\mathrm{S} / \mathrm{N}$ for the Apollo 12 sample (No. 12018.15) in the same manner but using the predicted

272 dipole moment as a signal. The Apollo sample (Fig. 3b) shows the signal amplitude of $B_{Y}=36.8 \mathrm{pT} \mathrm{rms}_{\mathrm{rms}}(104.5 \mathrm{pT}$ p-p) and

273 the noise of $28.4 \mathrm{pT}_{\mathrm{rms}}$, giving $\mathrm{S} / \mathrm{N}=1.30$ that is better $\mathrm{S} / \mathrm{N}$ than at the CEREGE laboratory. This is because (1) the

274 difference in the intensity of the magnetic moment and (2) the background at NASA vault is about $15 \%$ quieter than at

275 CEREGE. Therefore, we can estimate that the demagnetization experiment at CEREGE (Fig. 4b) was performed in

276 worse conditions than the operations that took place at LSLF, and that this test demonstrates that our magnetometer can

277 recover the signal from, at least, the condition $\mathrm{S} / \mathrm{N}=0.5$.

278 The detection limit for the magnetic moment can be defined by the point where the observed signal (in root mean 279 square amplitude) becomes equal to the standard deviation of noise. Figure 5 shows the estimation of the detection limit

280 for $B_{Y}$ at different noise floors at $\mathrm{S} / \mathrm{N}=1$. Since our magnetometer can adjust the sample to sensor distance $d$, the 281 sensitivity for the magnetic moment $m$ and the detection limit is a function of the distance and the noise floor. Because 282 our magnetometer can recover the signal from $\mathrm{S} / \mathrm{N}=0.5$ condition and the noise at NASA is $30 \mathrm{pT}_{\mathrm{rms}}$, we can measure 283 the magnetic moment above $15 \mathrm{pT}$ noise-floor line in the Fig.5. This figure also plots typical magnetic moment of three 284 major moon rock types at given weight, according to the previous study of the natural remanent magnetization (NRM) of 285 Apollo samples measured by SQUID ${ }^{6}$. At $d=20 \mathrm{~cm}$, we can measure most of breccia rocks down to $10 \mathrm{~g}$, whereas small 286 (several tens of grams) basalt rocks having slightly weaker NRM need to approach at $d=15 \mathrm{~cm}$. Even when the 287 background noise increases by a factor of 6 (90 pT line in Fig. 5), we can safely measure those types of rocks that have 288 relatively strong NRMs, if the sample is heavier than about $50 \mathrm{~g}$. Norite and anorthosite rocks, which are generally very 289 weakly magnetized, need a sensor-to-sample distance of about $d=10 \mathrm{~cm}$ to measure $>100 \mathrm{~g}$ samples, and even down to $290 d=5 \mathrm{~cm}$ for samples below $100 \mathrm{~g}$. With these detection limits, we could actually measure almost every breccia and 291 basalt sample in the Apollo collection, except those that are stored in steel containers.

292 In the equation (1), we consider only the sinusoidal output produced by a homogeneously magnetized spherical 293 sample that generate a dipole field ${ }^{19}$. However, assuming that the sample holder is completely filled by a sample and 294 homogeneously magnetized, such cubic sample does not generate a dipole magnetic field. To evaluate this shape effect, 295 we calculate the signal from a homogeneously magnetized cubic sample based on the calculation by Helbig ${ }^{23}$ in addition 296 to a dipole source (see Appendix B). Figure 6a shows the half-cycle of the calculated signals expressed as linkage 297 coefficients equivalent to $\boldsymbol{B} / \boldsymbol{m}$, showing M-shaped waveforms. The distance $(r)$ is equal to the length of the edge of the 298 cube $(a)$. The magnetic signal is reduced at the angles where the peaks of the dipole field are located $\left(\theta=0^{\circ}\right.$ for the radial component and $\theta=90^{\circ}$ for the tangential component), and the amount of error becomes maximum at these angles (Fig. 

shows the plots of the errors at $\theta=0^{\circ}\left(90^{\circ}\right)$ of the radial (tangential) components as functions of the normalized distance

$302(r / a)$. The error reduced rapidly with increasing the distance by a factor of $(r / a)^{-3.9}$. The normalized error becomes 303 acceptable (3.7\%) at $r / a=1.5$, ignorable (1\%) at $r / a=2.1$ and negligible $(0.26 \%)$ at $r / a=3$. Thus, as a rule of thumb, a 304 distance farther than $r / a \geq 1.5$ is recommended to reduce the shape effect. In the actual measurement of the Apollo 305 samples, $62 \%$ of the samples were measured at distance farther than $r / a=1.5$ and $93 \%$ of them were measured with $r / a \geq$ 3061.25 , based on the size of the cubic sample holder. Since the sample is always smaller than the holder, the actual $r / a$ ratio 307 is better than the value computed from the holder size. Therefore, we estimate that the deformation of the signal due to 308 the shape effect is small in our study. In fact, as mentioned in the previous section, most of the measured signals can be 309 explained by a dipole field. Detailed analyses of the harmonics will help us to reveal the origin of the heterogeneous 310 magnetizations ${ }^{17-20}$.

\section{CONCLUSION}

313 In order to measure the remanent magnetization of large bulk samples, we have developed a spinner magnetometer

314 equipped with a three-axis flux gate sensor and a large sample table enclosed within a two-layer mu-metal magnetic 315 shield resulting in a residual field of about $20 \mathrm{nT}$. The adjustable sensor position ( 5 to $30 \mathrm{~cm}$ ) enables the measurement of 316 small ( $5 \mathrm{~cm}$ cube) to large ( $20 \mathrm{~cm}$ cube) samples with acceptable deformation of the sinusoidal signals. By means of the 317 stacking technique of the signal, the experiments demonstrate that this instruments can measure weak (17 $\left.\mathrm{pT}_{\mathrm{rms}}\right)$ 318 sinusoidal signals for $\mathrm{S} / \mathrm{N}=0.5$. This performance indicates that the magnetometer can measure magnetic moments of 319 about $5 \times 10^{-7} \mathrm{Am}^{2}$ at the standard sample to sensor distance $d=15 \mathrm{~cm}$. This detection limit corresponds to the NRM of 320 about $10 \mathrm{~g}$ of lunar basalt or breccia. Because we focused on the samples that range from $22 \mathrm{~g}$ to $4.7 \mathrm{~kg}$ in mass, this 321 magnetometer can cover theoretically all of the basalt and breccia samples that we are interested in. We have already 322 conducted a first visit to NASA and measured 133 samples in 4 working days, demonstrating an optimized mechanism 323 and workflow of this magnetometer. In this study, we used the simplest magnetization model (single dipole source at the 324 stage center). However, due to the possible anisometric shape and/or off-center positioning in the cubes and/or 325 lithological heterogeneities, the actual sample may have off-centered and/or multiple dipole(s) that cannot be explained 326 by this simple magnetization model. In the future studies, we will customize the model for the individual samples by 327 integrating other information (e.g. shape and lithology) to explain the magnetic field distribution around such 

samples, e.g. whole meteorites and archeological artifacts, without destructive sampling.

\section{ACKNOWLEDGEMENT}

This project was made possible by a seed funding from the Programme National de Planétologie (INSU-CNES), and subsequent funding by the Agence Nationale de la Recherche (grant ANR-14-CE33-0012). We are greatly indebted to the lunar sample curators and processors (Ryan Zeigler, Andrea B. Mosie, Darvon Collins, Anthony Ferrell) at NASA Johnson Space Center for their time, patience and understanding. J.G. acknowledges funding from People Programme (Marie Curie Actions) of the European Union's Seventh Framework Programme (FP7/2077-2013) under REA grant agreement no. 298355. EAL would like to thank NASA grants NNA14AB01A and NNX15AL62G and NSF grant DMS1521765 for partial support. M.U. wishes to thank Ateliers Mécaniques de Précision in Eguilles for their fine mechanical processing.

\section{APPENDIX A}

The eq. (1) indicates that the radial and tangential component of a dipole moment can be observed as a sinusoidal curve given by $B(\theta)=A \sin (\theta)$. When we use an encoder with a resolution of $N$ positions per revolution, the encoder resolution is $\Delta \theta=2 \pi / N$ and the apparent encoder angle is $\theta_{\text {enc }}(n)=\mathrm{n} \times \Delta \theta=2 \pi n / N$. According to eq. (2) the worst-case signal error (quantization error) for the observation of $B(\theta)$ at $\mathrm{n}$-th encoder position becomes

$$
\operatorname{err}(\theta, \Delta \theta)=B\left(\theta+\theta_{\text {err }}\right)-B(\theta)=\Delta \theta \times a\left(\theta_{\text {enc }}(n)\right)=\Delta \theta \times \frac{d(A \sin (\theta))}{d \theta}(\mathrm{n} \Delta \theta)=\Delta \theta A \cos (\mathrm{n} \Delta \theta)(A 1) .
$$

The absolute value of this signal error becomes maximum of $A \times \Delta \theta=2 \pi A / \mathrm{N}$ when $|\cos (\mathrm{n} \Delta \theta)|=1$.

Using the averaging technique, we average the signal between $n$-th and $(n+1)$-th encoder position to represent the magnetic field when $\theta$ is in range of $\theta_{\text {enc }}(n) \leq \theta<\theta_{\text {enc }}(n+1)$. The stacking technique increases number of measurement to be averaged. The averaged signal at this $\theta$ is given by

For a large enough number $N$, we can use $\sin (\Delta \theta / 2) \sim \Delta \theta / 2$ and to approximate this integration,

$$
\bar{B}(\theta)=\frac{1}{\Delta \theta} \int_{n \Delta \theta}^{(n+1) \Delta \theta} A \sin (\theta) d \theta \quad(A 2)
$$


case error for $\theta_{\text {enc }}(n)$ is given by

$$
\begin{gathered}
\bar{B}(\theta)-B\left(\theta_{\text {enc }}(n)\right)=A \sin \left(n \Delta \theta+\frac{\Delta \theta}{2}\right)-A \sin (n \Delta \theta) \\
=2 A \sin \left(\frac{\Delta \theta}{4}\right) \cos \left(n \Delta \theta+\frac{\Delta \theta}{4}\right) \approx \frac{\Delta \theta}{2} A \cos \left(\left(n+\frac{1}{4}\right) \Delta \theta\right) \quad(A 4) .
\end{gathered}
$$

The worst-case signal error is, therefore, approximately half of the no-averaging case given by eq. (A1).

A similar error can occur due to the second ordered low-pass filter with a cut-off frequency of $100 \mathrm{~Hz}$ built-in in the signal conditioning unit when the rotation speed is too fast. We suppose that the error of the low-pass filter can be acceptable $(86.5 \%$ of the final value) after $2 \tau \mathrm{s}$, where $\tau$ is the time constant of this filter (about $2.5 \mathrm{~ms}$ ). The sample rotates $2 \tau v^{\circ}$ when the rotation speed is given by $v \%$ shen the error diminished to an acceptable amplitude. Thus, if the resolution of the encoder $\left(\Delta \theta=360^{\circ} / N\right)$ is smaller than $2 \tau v$, the effect of the low-pass filter is not observable. Such critical rotation speed $v_{c}=\Delta \theta / 2 \tau=360 /(2 N \tau)=141 \%$ s. According to our measurement of instantaneous rotation speed in the actual measurement, we rotated the sample generally slower than $v_{c}$. However, for short periods the rotation speed sometimes reaches up to $2 v_{c}$. This make a similar effect from the quantization error discussed above, resulting error of (1 - $\exp (-2)) \times \operatorname{err}(\theta, \Delta \theta \times$ floor $(2 \tau v / \Delta \theta))=0.135 \times \Delta \theta \times$ floor $(2 \tau v / \Delta \theta)$ using eq. (A1). The function floor $(x)$ returns the integer part of $x$. This error is $0.135 \times 2 \Delta \theta$ when $v=2 v_{c}$. Thus, the estimated error due to the low pass filter is about $27 \%$ of the quantization error, which can be ignored. This error becomes comparable to the quantization error when $v$ becomes $7.4 v_{c}=1044 \%$, which is equivalent to $2.9 \mathrm{~Hz}$ sample rotation frequency, for our combination of low-pass filter $(\tau=2.5$ $\mathrm{ms})$ and encoder $(N=512)$. This is five times faster than the actual rotation speed. Therefore, the low-pass filter with cutoff frequency of $100 \mathrm{~Hz}$ used in our system does not modify the waveform of the signal from the sample.

\section{APPENDIX B}

We calculated the magnetic field around homogeneously magnetized isotropic (spherical) and cubic samples using the linkage tensor between a homogeneously magnetized body and the magnetic field given by Helbig (1965). The linkage tensor can be regarded as a normalized, dimensionless magnetic field intensity. We assumed a magnetization moment directed to $+\mathrm{x}$ and located at the origin of a three dimensional Cartesian coordinate system. We considered the distribution of the magnetic field in the $\mathrm{x}$-y plane. According to Helbig (1965), at the position $(x, y, z)$, the distance from the dipole $(u, v, w)=(x-0, y-0, z-0)$ and the linkage tensors for the dipole field generated by an isotropic body are given by

$$
g_{x x}^{d}=\frac{2 u^{2}-v^{2}-w^{2}}{e^{5}}
$$


and

$$
g_{x y}^{d}=\frac{3 u v}{e^{5}} \quad(\mathrm{~B} 1)
$$

where the superscript $d$ indicated the dipole, the subscript $x x$ and $x y$ respectively indicates the contribution of the $+x$ directed magnetization to the $x$ and $y$ components of the magnetic field, and $e^{2}=u^{2}+v^{2}+w^{2}$.

The three fold integration of (B1) yield the linkage tensors for a cubic sample, which has been already given by the equations (4) in Helbig (1965). Some calculated values in the first quadrant has been given in Table 1 of Helbig (1965).

394 However, the equations of Helbig (1965) do not reproduce the calculated values; they also cannot be applied to our calculation directly due to some problems. We used the equation modified after equations (4) in Helbig (1965),

and

$$
g_{x x}=\left[\left[\left[\frac{u}{|u|} \arcsin \left(\frac{v \cdot w}{\sqrt{u^{2}+v^{2}} \cdot \sqrt{u^{2}+w^{2}}}\right)\right]_{x+a / 2}^{x-a / 2}\right]_{y+b / 2}^{y-b / 2}\right]_{z+c / 2}^{z-c / 2}
$$

$$
g_{x y}=\left[\left[\left[\ln \left(\frac{w+e}{u^{2}+v^{2}}\right)\right]_{x+a / 2}^{x-a / 2}\right]_{y+b / 2}^{y-b / 2}\right]_{z+c / 2}^{z-c / 2} \quad(\mathrm{~B} 2),
$$

where $a, b$, and $c$ are the length of the sides parallel to the $\mathrm{x}-, \mathrm{y}-$, and z-axis, respectively. The added term $u /|u|$ in $g_{x x}$ gives the sign of $u$ to extend the function to other quadrants. Note that $(u, v, w)=(x, y, z)$ for the moment placed at the origin. The absolute value of $w$ in $g_{x y}$, which can be found in the original equation, is typographical error, since the equation replaced $|w|$ with $w$ successfully reproduces the calculation results given in the Table 1 of Helbig (1965). Finally, the magnetic fields can be expressed in polar coordinates by $g_{x r}(r, \theta)=g_{x x}(x, y, z) \times \cos (\theta)+g_{x y}(x, y, z) \times \cos (\theta)$ and $g_{x t}(r, \theta)$

$404=g_{x y}(x, y, z) \times \cos (\theta)-g_{x x}(x, y, z) \times \sin (\theta)$, which respectively indicates the radial and tangential contributions at the position $(x, y, z)=(r \times \cos (\theta), r \times \sin (\theta), 0)$. The calculation has been conducted with Maxima (http://maxima.sourceforge.net).

\section{REFERENCES}

$415{ }^{1}$ M. E. Purucker and J. B. Nicholas, Journal of Geophysical Research: Planets 115, E12007 (2010).

$416{ }^{2}$ H. Tsunakawa, H. Shibuya, F. Takahashi, H. Shimizu, M. Matsushima, A. Matsuoka, S. Nakazawa,

${ }^{4}$ B. P. Weiss and S. M. Tikoo, Science 346, 1246753 (2014). 
${ }^{5}$ I. Garrick-Bethell, B. P. Weiss, D. L. Shuster and J. Buz, Science 323, 356 (2009). (2012).

$425{ }^{8}$ S. M. Tikoo, B. P. Weiss, W. S. Cassata, D. L. Shuster, J. Gattacceca, E. A. Lima, C. Suavet, F. 426 Nimmo and M. D. Fuller, Earth and Planetary Science Letters 404, 89 (2014).

427 9J. Buz, B. P. Weiss, S. M. Tikoo, D. L. Shuster, J. Gattacceca and T. L. Grove, Journal of 428 Geophysical Research: Planets 120, 1720 (2015).

${ }^{10}$ W. S. Goree and M. Fuller, Reviews of Geophysics 14, 591 (1976).

$430{ }^{11}$ J. L. Kirschvink, R. E. Kopp, T. D. Raub, C. T. Baumgartner and J. W. Holt, Geochemistry, 431 Geophysics, Geosystems 9, Q05Y01 (2008).

$432{ }^{12}$ T. A. T. Mullender, T. Frederichs, C. Hilgenfeldt, L. V. de Groot, K. Fabian and M. J. Dekkers, 433 Geochemistry, Geophysics, Geosystems 17, 3546 (2016).

$434{ }^{13}$ G. Pearce, W. Gose and D. Strangway, Proceedings of the Lunar Science Conference (supplement 435 4, Geochimica et Cosmochimica Acta) 3, 3045 (1973).

$436{ }^{14}$ H. Wang and B. P. Weiss, AGU meeting abstract \#167372 (2016).

$437{ }^{15}$ M. Funaki, M. Koshita and H. Nagai, Antarctic Meteorite Research 16, 220 (2003).

$438{ }^{16}$ E. Thellier, Methods in Palaeomagnetism, edited by D. W. Collinson, K. M. Creer and S. K. 439 Runcorn (Elsevier Amsterdam, 1967).

$440 \quad{ }^{17}$ L. Molyneux, Geophysical Journal International 24, 429 (1971).

$441{ }^{18}$ M. Kono, Y. Hamano, T. Nishitani and T. Tosha, Geophysical Journal International 67, 217 442 (1981).

$443 \quad{ }^{19}$ D. W. Collinson, Reviews of Geophysics 13, 659 (1975). 
$444{ }^{20}$ K. Kodama, Geochemistry, Geophysics, Geosystems 18, 434 (2017).

$445{ }^{21}$ M. Uehara, J. Gattacceca, P. Rochette, F. Demory and E. M. Valenzuela, Physics of the Earth and 446 Planetary Interiors 200-201, 113 (2012).

$447{ }^{22}$ R. Fisher, Proceedings of the Royal Society of London. Series A. Mathematical and Physical 448 Sciences 217, 295 (1953).

$449{ }^{23}$ K. Helbig, Journal of geomagnetism and geoelectricity 17, 373 (1965). 
(a)

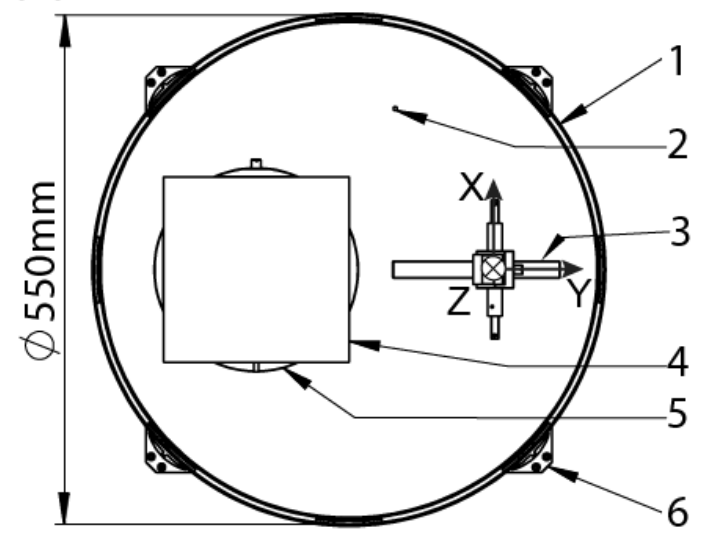

(b)

FIG. 1. Schematic illustrations of the magnetometer in the top view opening the top cover (a) and the side view showing the interior by a broken-out section of the shield (b). A two-layered mu-metal shield (1) enclosing a three-axis fluxgate sensor (7) mounted on a sensor holder (8) that can slide on a rail (3), and a sample (4) on a rotating table (5). The sensor-to-sample distance is adjusted by changing the position of the pin (9) fixed on bores (10). The user can rotate the table by a handle (14) and the rotation angle is measured by an optical encoder unit (13). The power supply and the outputs of the sensor are connected to the outer signal conditioning unit via feedthroughs (2) and a connector (12). The mu-metal shield and the entire system is mounted on an aluminum plate (6) supported by aluminum feet (11). The coordinate system is shown in the figures. The horizontal and vertical lines in (b) show rotation axis and the horizontal centerline of the sensor, respectively. 


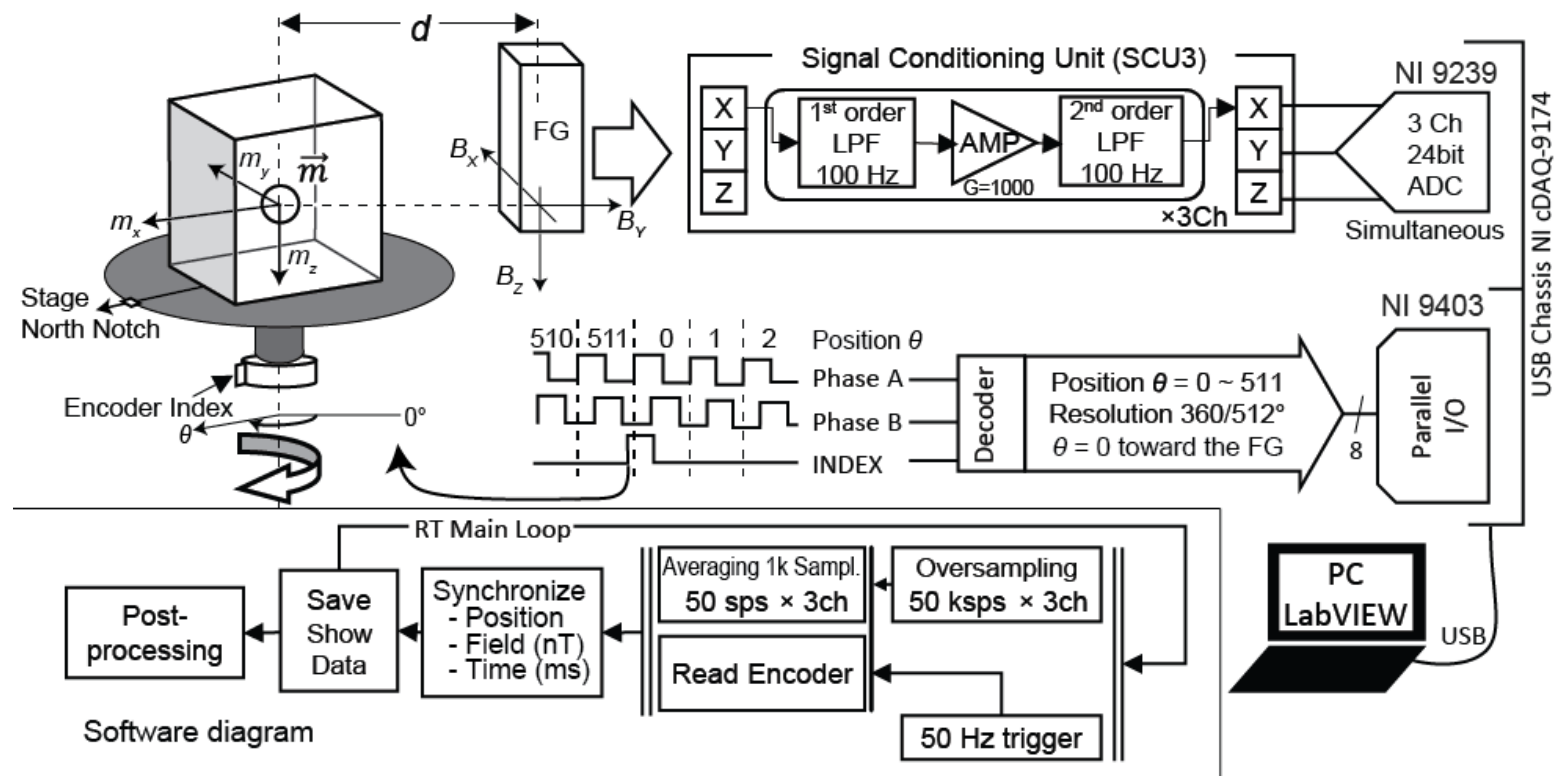

FIG. 2. Schematic diagrams of the magnetometer. The magnetic field from the magnetic moment of the sample $(\overrightarrow{\boldsymbol{m}})$ is detected by the 3 axis fluxgate sensor (FG) at the distance $d$ connected to the signal conditioning unit (SCU3) that filters high frequency noises and amplitude at a gain of 1000 . The output of the three magnetic field components $\left(B_{X}, B_{Y}, B_{Z}\right)$ are simultaneously digitized by 3 channels of a 24-bit A/D converter (NI9239). The encoded rotation angle of the sample table $(\theta)$ is decoded by a decoder IC connected to a parallel I/O unit (NI 9403) and converted in a relative angle. The zero position is where the index of the encoder exactly faces the fluxgate sensor. The resolution is 512 steps per a revolution. The A/D unit and the I/O unit are mounted to a USB chassis (NI cDAQ9174) and connected to a PC via USB port. A Lab-VIEW program controls the quasi real-time routine (RT Main Loop) and treats the data every $20 \mathrm{~ms}(50 \mathrm{~Hz})$. 
(a)

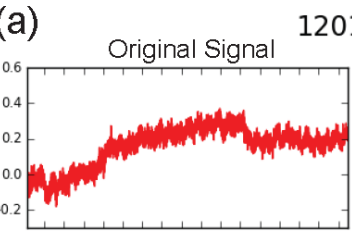

12018.15
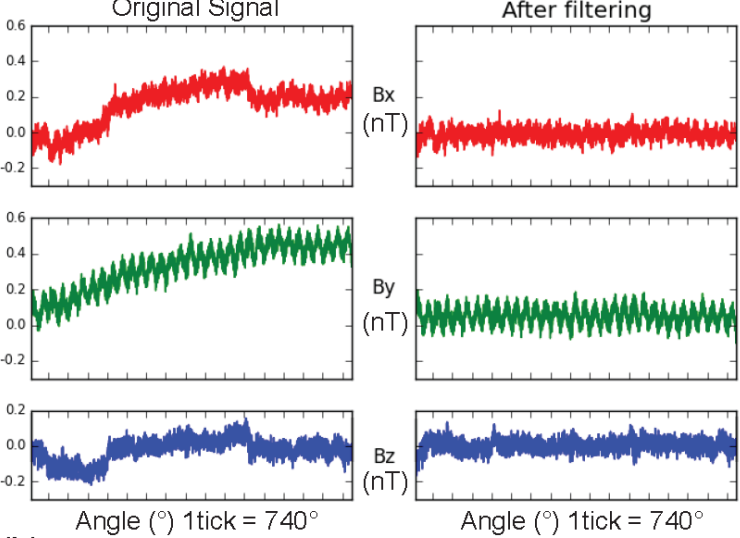

(b)
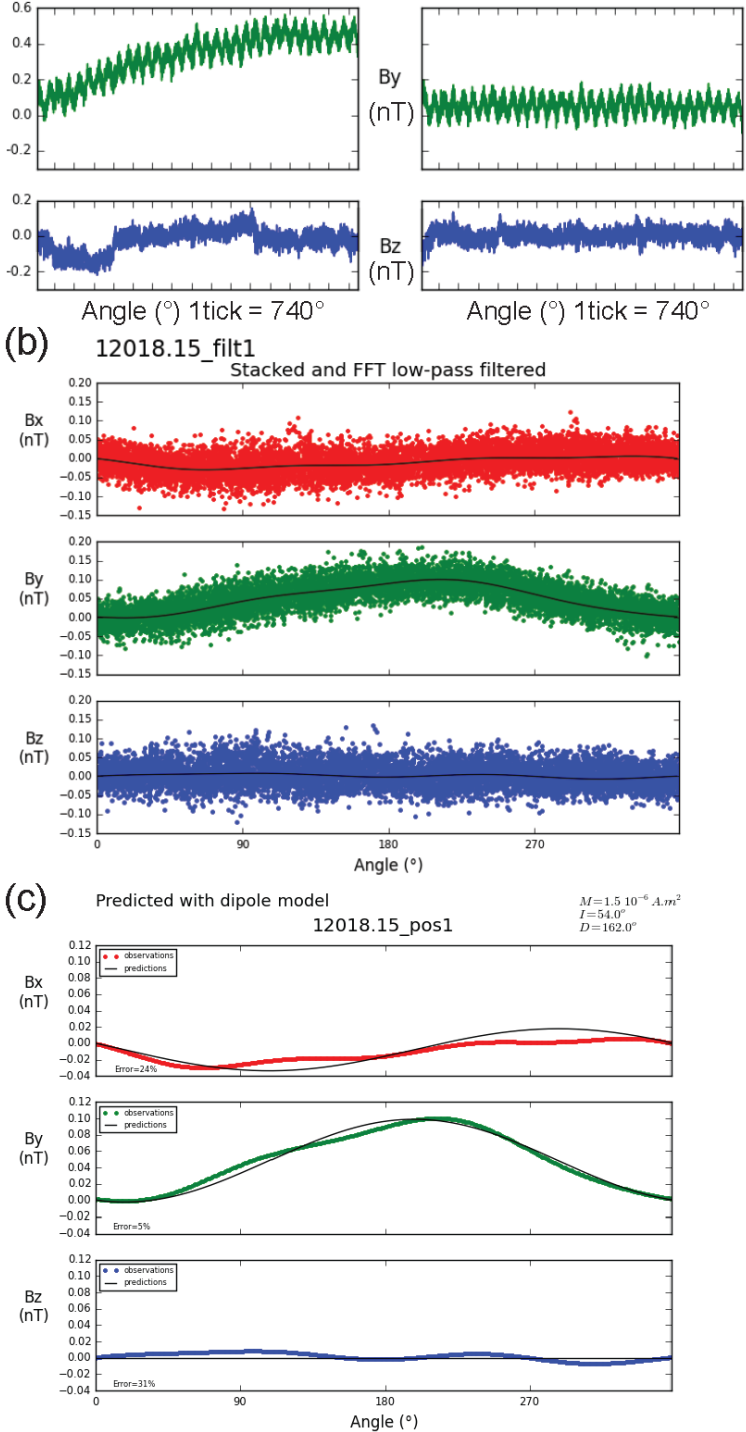

FIG. 3. An example of signal treatment procedure. The sample No. 12018.15, which was collected by the Apollo 12 mission, is one of the most weakly magnetized samples. The magnetic field is measured at the position 1, observed at $d=15 \mathrm{~cm}$, and rotated 32 revolutions. All magnetic field intensities are relative to the initial value. (a)The first step is the drift and jump correction by a highpass filter. The original signal converted in $\mathrm{nT}$ and plotted as a distribution over the absolute rotation angle (right chart). The small ripples having wavelength of $360^{\circ}$ corresponding to sinusoidal signals generated by revolutions of the sample. Large drifting (400 pT) and jumping (100 pT) are observable, which have been removed by the filtering (left chart). (b) The stacked data (solid dots) compiled for a single revolution $\left(360^{\circ}\right)$ and its FFT low-pass filtered result (solid lines) after drift and jump corrections. (c) A prediction (solid black lines) with a dipole model after an inversion calculation involving data obtained at other two positions (position 2 and 3 ). The predicted dipole is $m=1.5 \times 10^{-6} \mathrm{Am}^{2}, I=-54^{\circ}$, and $D=162^{\circ}$. 
(a)

AF Demag - StdBlockNo13 $(w=0.98 \mathrm{~g})$

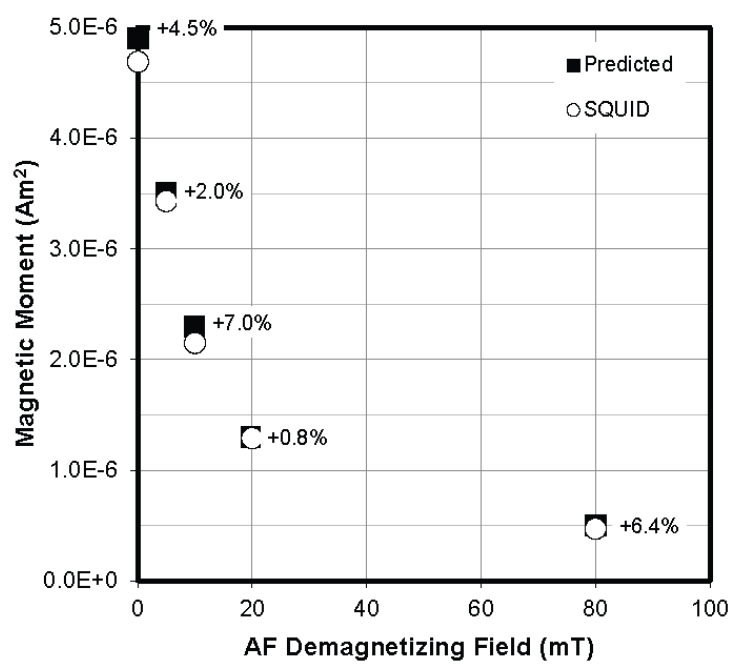

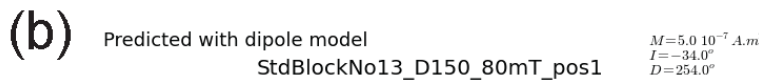
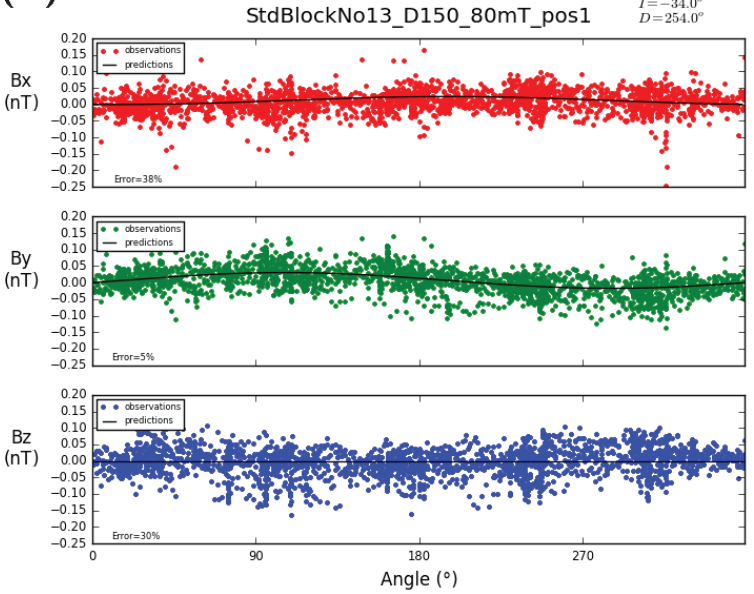

FIG. 4. A demonstration of the magnetometer performances using a standard sample that is a small $(0.98 \mathrm{~g})$ fragment of a basalt rock. (a) The standard sample is demagnetized by alternating magnetic field (AF) up to $80 \mathrm{mT}$ and measured by the developed spinner magnetometer (solid square symbols, after prediction using 3 positions) and the SQUID magnetometer (open circle symbols). The error of the prediction is also shown in percent of the SQUID results. Our data predicts the actual dipole moment measured by SQUID magnetometer in $0.8 \%$ to $7.0 \%$ of overestimation. (b) The observations after data treatments at position 1 (dots) and the predictions of a dipole model using three positions (black lines) at $80 \mathrm{mT} \mathrm{AF} \mathrm{demagnetization} \mathrm{step.} \mathrm{The} \mathrm{predicted} \mathrm{dipole} \mathrm{moment} \mathrm{is} m=5.0 \times 10^{-7}$ $\mathrm{Am}^{2}, I=34^{\circ}$, and $D=254^{\circ}$. 


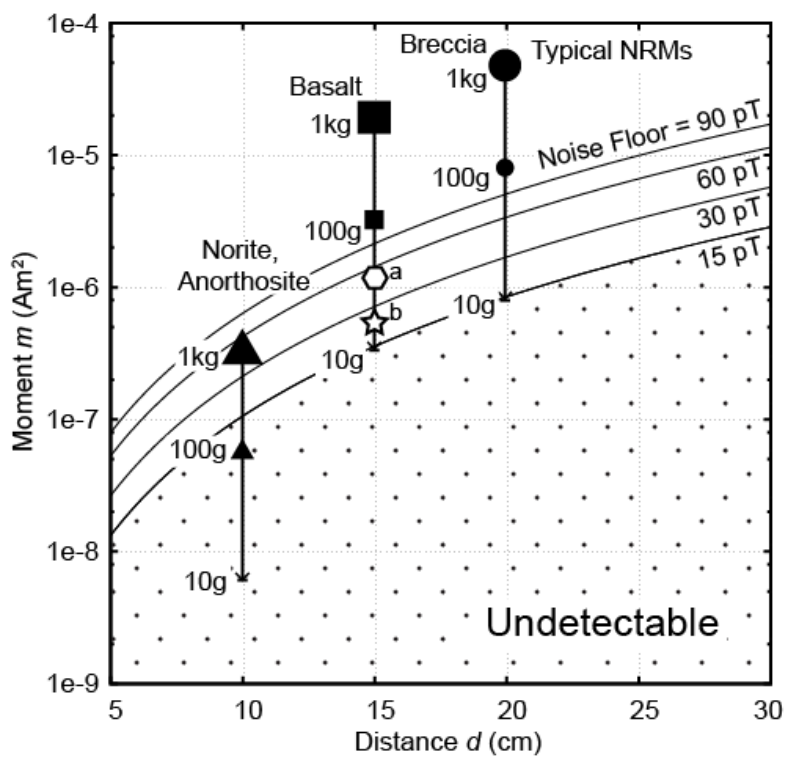

FIG. 5. Detection limits of the magnetometer for different sensor noise floors (15 pT, $30 \mathrm{pT}, 60 \mathrm{pT}$, and $90 \mathrm{pT})$. The solid lines indicate where the peak-to-peak intensity of the sinusoidal signal by a rotation of a dipole moment $m$ observed at distance $d$ becomes equal to the given noise intensity. Note that the inclination of the dipole is horizontal making the largest amplitudes. The magnetic moment at the hatched region is undetectable due to the weak signal below noise floor of the sensor. The calculated intensities of natural remanent magnetizations (NRMs) of different masses and types of moon rocks are also shown. The NRMs of the moon rocks are given by a previous study ${ }^{6}$. The samples measured in this study are also shown $(\mathrm{a}=12018.15$ at NASA, $\mathrm{b}=$ StdBlockNo13 at CEREGE; measurements data shown in figures 3 and 4 , respectively). 

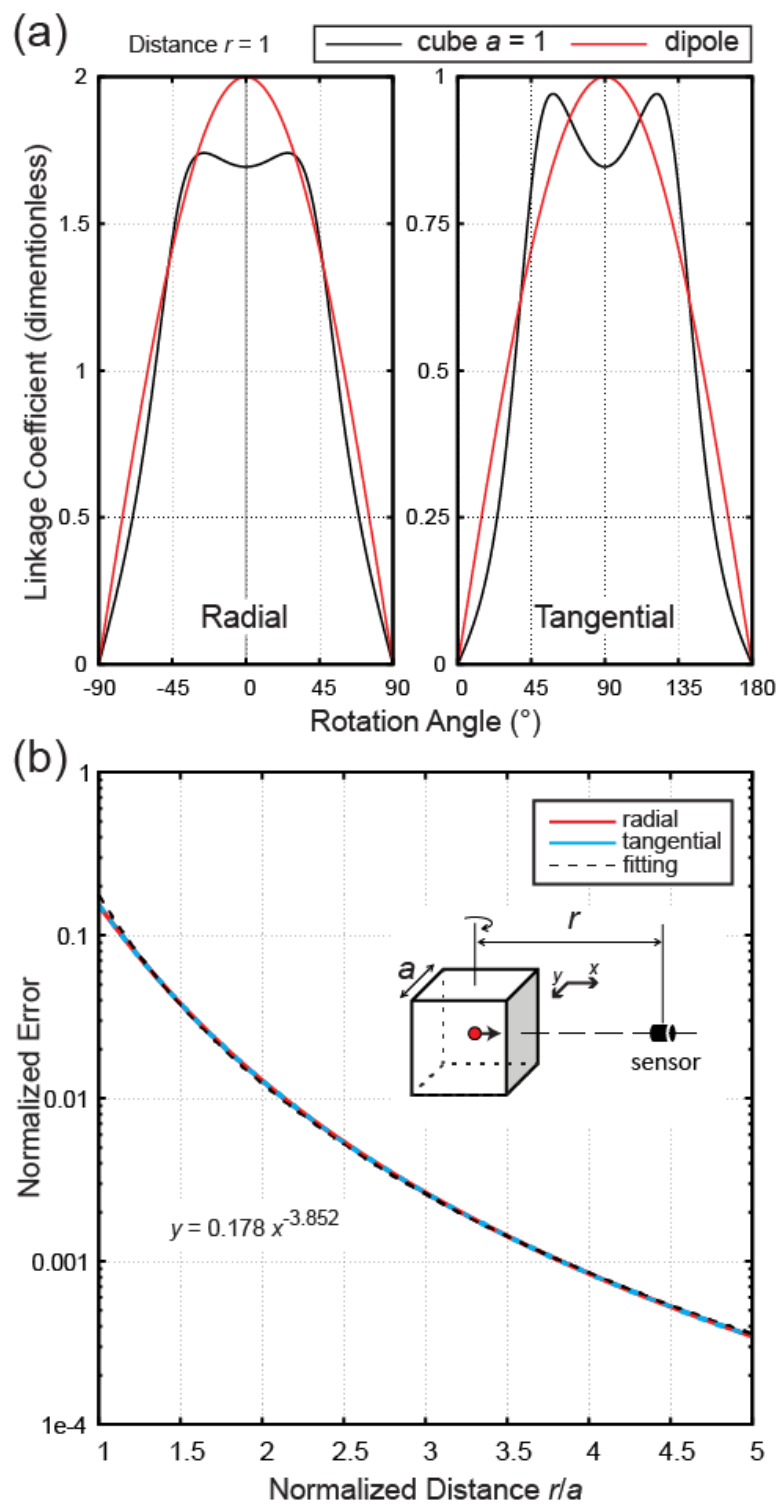

FIG. 6. (a) The half-cycle of the calculated signals according to Helbig ${ }^{23}$, expressed as linkage coefficient for the radial and tangential components. The distance $(r)$ equals to the length of the edge of the cube $(a)$. The sinusoidal curves indicate the signal from dipole source and the M-shaped deformed curve is the signal from a cubic homogeneously magnetized sample. The difference between the signal from the cube and the dipole source becomes maximum at the peak of the sinusoidal curve $\left(0^{\circ}\right.$ for the radial component and $90^{\circ}$ for the tangential component). (b)The maximum error normalized by the amplitude of the dipole field (normalized error) as a function of the distance normalized by the length of the edge $(r / a)$. A fitting curve is also shown. The inset of (b) shows the geometry of the samples and the sensor. 

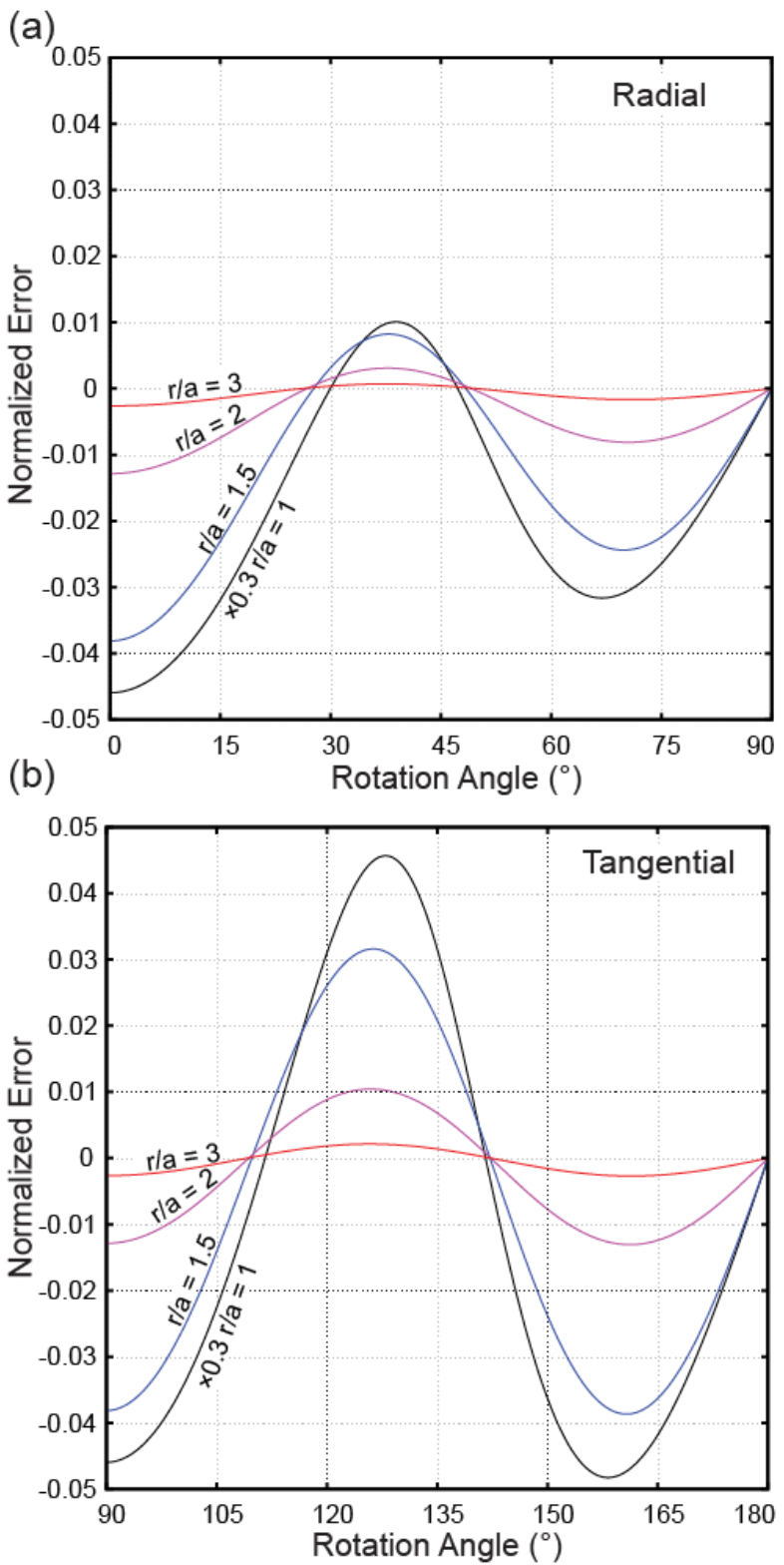

FIG. 7.The errors due to the shape effect of a cubic-shaped sample at different normalized sensor-to-sample distances $(r / a)$.The ranges of the rotation angle are limited to quarter cycles from the peak position of the dipole field $\left(0^{\circ}\right.$ for the radial component, $\mathrm{a} ; 90^{\circ}$ for the tangential component, b). The values are normalized by the amplitude of the dipole field. The curves for $r / a=1$ is reduced to 5140.3 of the original curves. 
TABLE 1. Alternating field (AF) demagnetization result of StdBlockNo13, showing intensity, declination, and inclination obtained by the prediction given by the inversion results of the developed spinner magnetometer and the observation by the SQUID magnetometer. The intensity and angular errors (|Dir|) between the predictions and the SQUID vector moments are also shown. The angular error is in absolute values.

\begin{tabular}{ccccccccc}
\hline \hline \multirow{2}{*}{$\begin{array}{c}\text { AF Field } \\
(\mathrm{mT})\end{array}$} & \multicolumn{3}{c}{ Prediction } & \multicolumn{3}{c}{ Observation SQUID } & \multicolumn{2}{c}{ Error } \\
\cline { 2 - 9 } & $\operatorname{Int}\left(\mathrm{Am}^{2}\right)$ & $\operatorname{Dec}\left({ }^{\circ}\right)$ & $\operatorname{Inc}\left(^{\circ}\right)$ & $\operatorname{Int}\left(\mathrm{Am}^{2}\right)$ & $\operatorname{Dec}\left({ }^{\circ}\right)$ & $\operatorname{Inc}\left({ }^{\circ}\right)$ & $\operatorname{Int}\left(\mathrm{Am}^{2}\right)$ & $|\operatorname{Dir}|\left(^{\circ}\right)$ \\
\hline 0 & $4.90 \mathrm{E}-06$ & 200 & 40 & $4.69 \mathrm{E}-06$ & 201 & 37 & $2.09 \mathrm{E}-07$ & 3.5 \\
5 & $3.50 \mathrm{E}-06$ & 200 & 46 & $3.44 \mathrm{E}-06$ & 198 & 44 & $6.47 \mathrm{E}-08$ & 2.6 \\
10 & $2.30 \mathrm{E}-06$ & 208 & 44 & $2.15 \mathrm{E}-06$ & 211 & 42 & $1.53 \mathrm{E}-07$ & 2.6 \\
20 & $1.30 \mathrm{E}-06$ & 238 & 42 & $1.29 \mathrm{E}-06$ & 229 & 40 & $1.30 \mathrm{E}-08$ & 7.3 \\
80 & $5.00 \mathrm{E}-07$ & 254 & 34 & $4.70 \mathrm{E}-07$ & 242 & 31 & $3.03 \mathrm{E}-08$ & 10.4 \\
\hline \hline
\end{tabular}

TABLE 2. A result of repeated measure of sample StdBlockNo13, showing intensity, declination, and inclination obtained by the prediction given by the inversion results. The mean value, the standard deviation, the semi-angle of aperture of the $95 \%$ confidence cone $\left(\alpha_{95}\right)$ and the angular standard deviation $\left(\theta_{65}\right)$ of the magnetic moment vectors are also shown.

\begin{tabular}{cccc}
\hline \hline \#Run & Int $\left(\mathrm{Am}^{2}\right)$ & Dec $\left(^{\circ}\right)$ & $\operatorname{Inc}\left(^{\circ}\right)$ \\
\hline 1 & $8.95 \mathrm{E}-05$ & 2.5 & 1.5 \\
2 & $9.37 \mathrm{E}-05$ & 0.1 & 2.3 \\
3 & $9.61 \mathrm{E}-05$ & 1.4 & 0 \\
4 & $9.53 \mathrm{E}-05$ & 0.6 & 2.1 \\
5 & $9.39 \mathrm{E}-05$ & 0.7 & 3.8
\end{tabular}

\begin{tabular}{|c|c|c|c|}
\hline Mean & $9.37 \mathrm{E}-05$ & 1.1 & 1.9 \\
\hline Stdev & $2.56 \mathrm{E}-06$ & - & - \\
\hline$\alpha_{95}$ & - & & \\
\hline$\theta_{65}$ & - & & \\
\hline
\end{tabular}

TABLE 3. Result of measurement of sample StdBlockNo13 at different sensor-to-sample distance, showing intensity, declination, and inclination obtained by the prediction given by the inversion results. The mean value, the standard deviation, the semi-angle of aperture of the $95 \%$ confidence cone $\left(\alpha_{95}\right)$ and the angular standard deviation $\left(\theta_{65}\right)$ of the magnetic moment vectors are also shown.

\begin{tabular}{cccc}
\hline \hline Distance $(\mathrm{mm})$ & Int $\left(\mathrm{Am}^{2}\right)$ & Dec $\left(^{\circ}\right)$ & Inc $\left(^{\circ}\right)$ \\
\hline 80 & $9.47 \mathrm{E}-05$ & -0.5 & -91.3 \\
100 & $9.29 \mathrm{E}-05$ & 0.1 & -91.8 \\
130 & $9.54 \mathrm{E}-05$ & 1.1 & -91.6 \\
160 & $1.01 \mathrm{E}-04$ & 1.8 & -94.0 \\
& & & \\
\hline Mean & $9.59 \mathrm{E}-05$ & 0.5 & -92.4 \\
Stdev & $3.36 \mathrm{E}-06$ & - & - \\
$\alpha 95$ & - & \multicolumn{2}{c}{$1.7^{\circ}$} \\
$\theta_{65}$ & - & \multicolumn{2}{c}{$2.2^{\circ}$} \\
\hline \hline
\end{tabular}


(a)

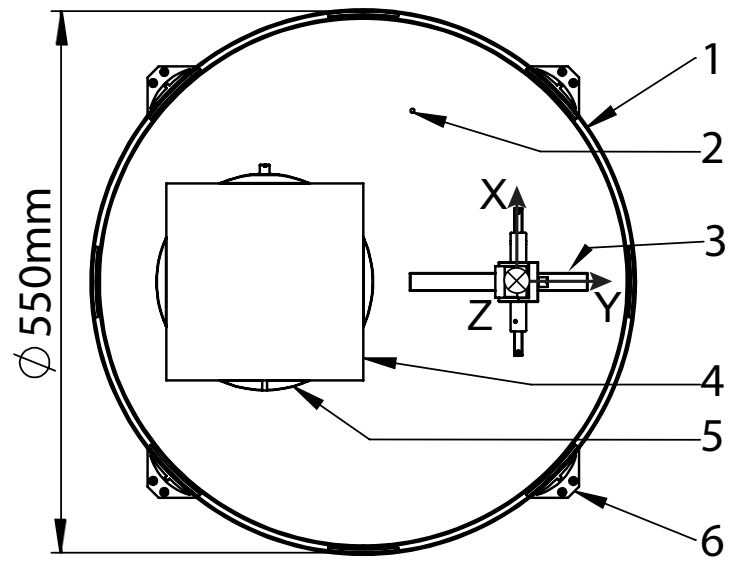

(b)

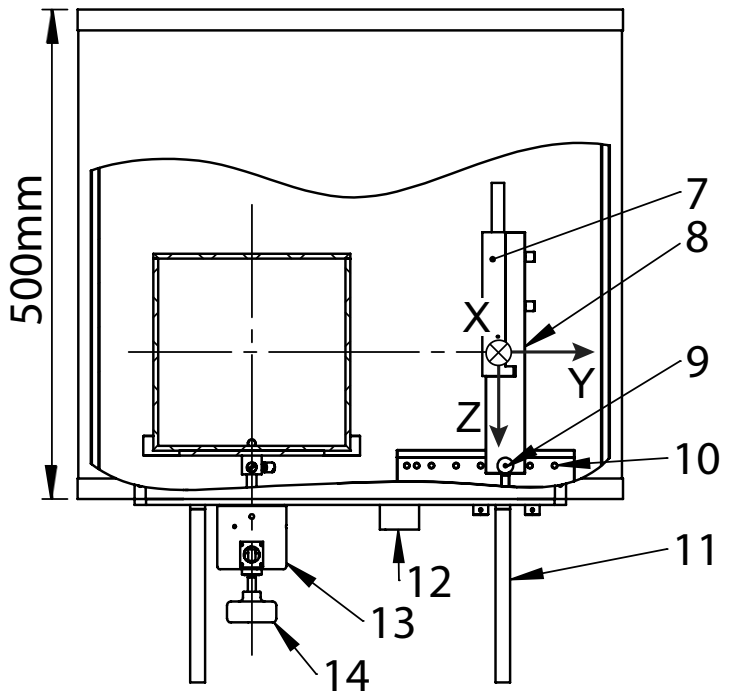


Stage

North Notch

Encoder Index
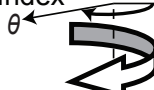

$\theta \longleftarrow$

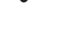

$d$
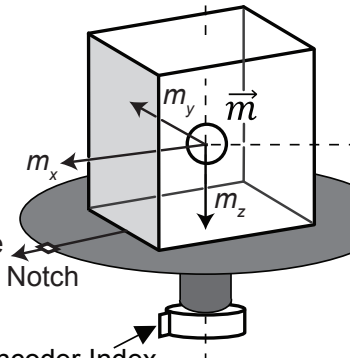

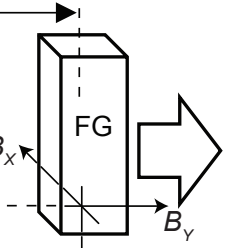

$B_{z} \downarrow$

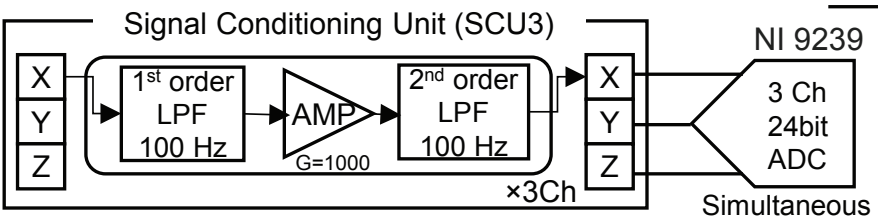

Simultaneous

510:511: $0: 1: \begin{array}{llll} & 0 & \text { Position } \theta\end{array}$

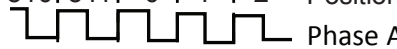
口أl INDEX

\section{RT Main Loop}

RT Main Loop

\begin{tabular}{|c|}
\hline $\begin{array}{c}\text { Post- } \\
\text { processing }\end{array}$ \\
$\begin{array}{l}\text { Save } \\
\text { Show } \\
\text { Data }\end{array}$ \\
\hline
\end{tabular}

Software diagram

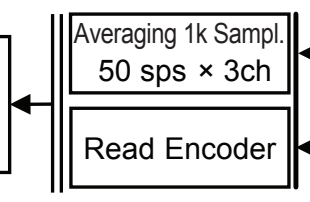

$50 \mathrm{ksps} \times 3 \mathrm{ch}$

Position $\theta=0 \sim 511$

Resolution 360/512 ${ }^{\circ}$

$\theta=0$ toward the $F G$ 


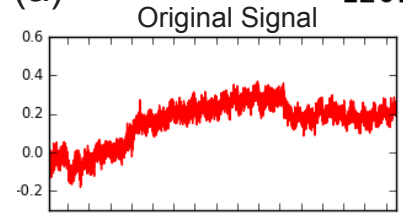

After filtering

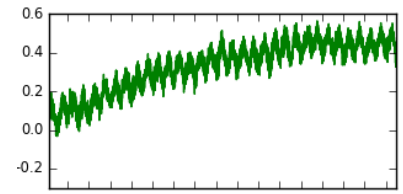

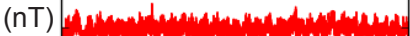

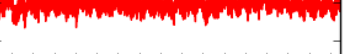

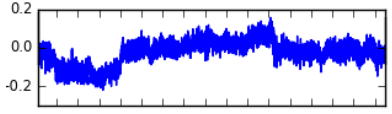

Angle $\left({ }^{\circ}\right) 1$ tick $=740^{\circ}$

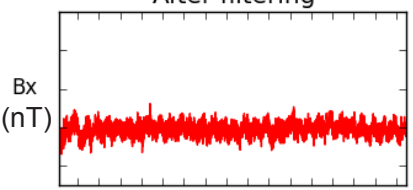

(b) 12018.15_filt1
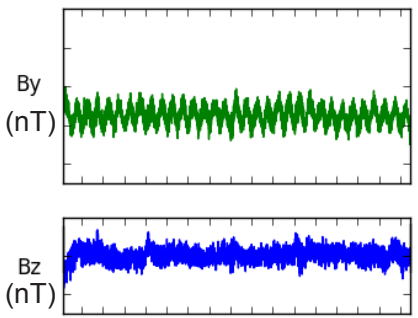

Angle $\left({ }^{\circ}\right) 1$ tick $=740^{\circ}$
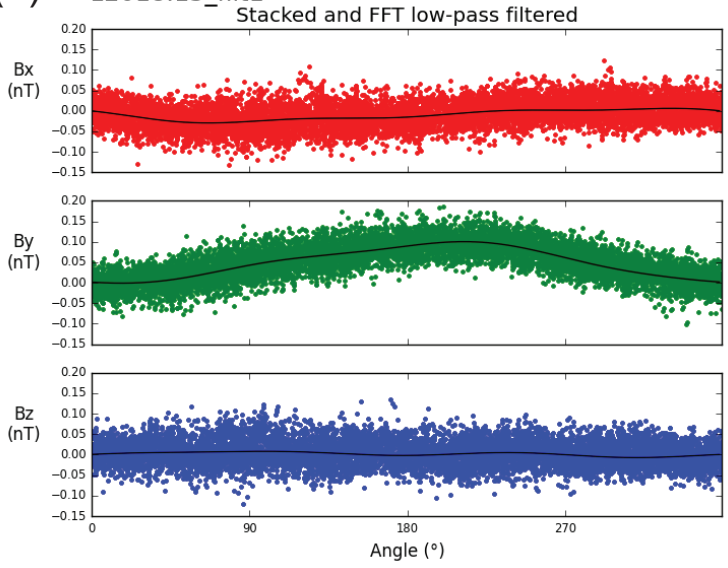

(c)
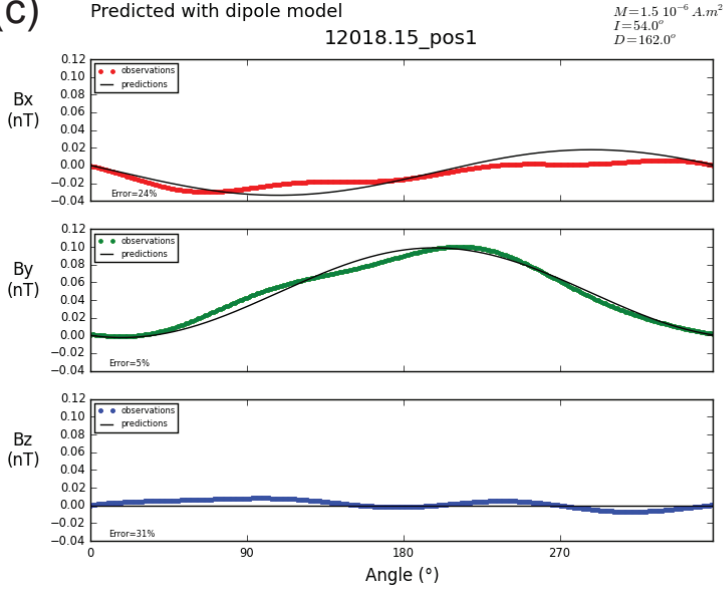
AF Demag - StdBlockNo13 ( $w=0.98 \mathrm{~g})$

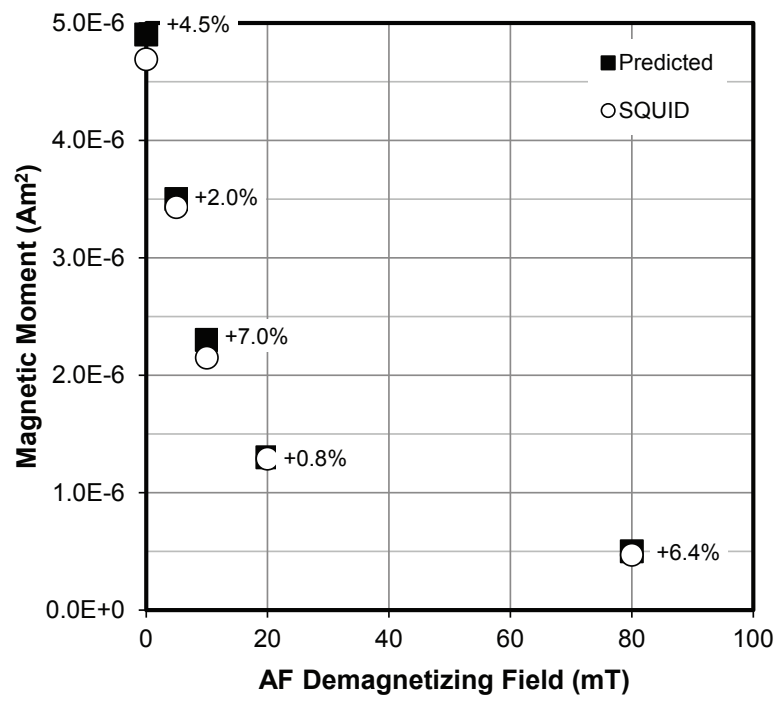

(D) Predicted with dipole model

StdBlockNo13_D150_80mT_pos1
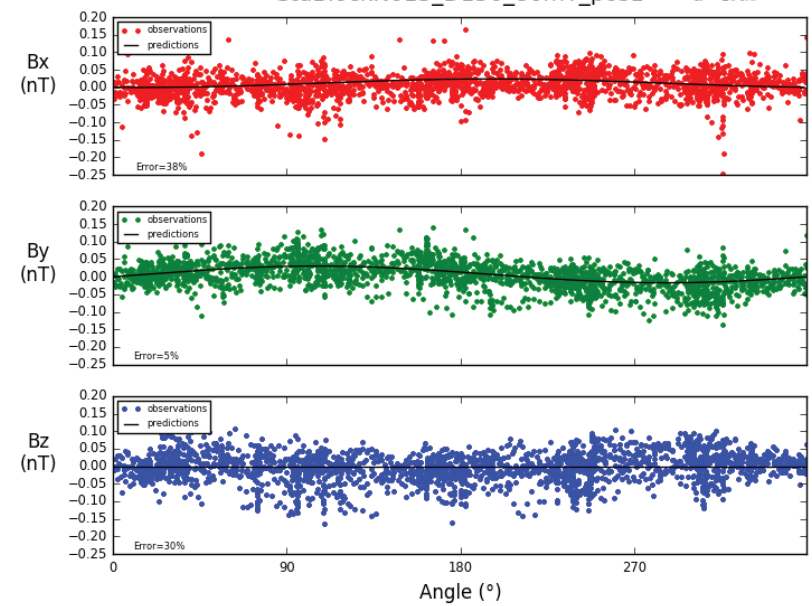


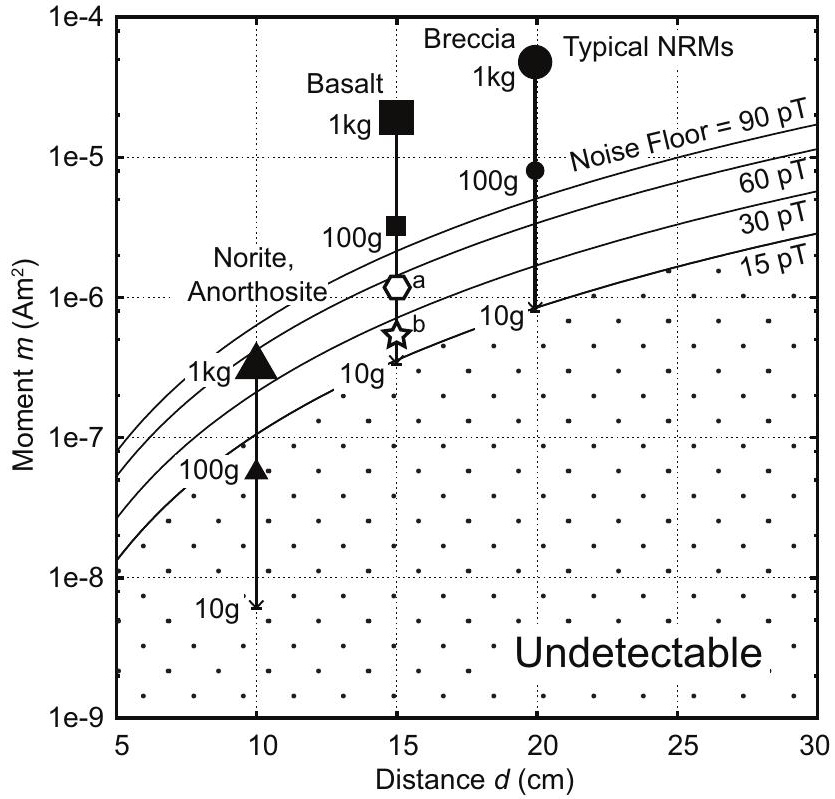



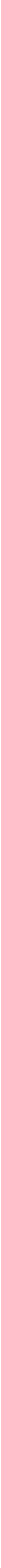
(a)
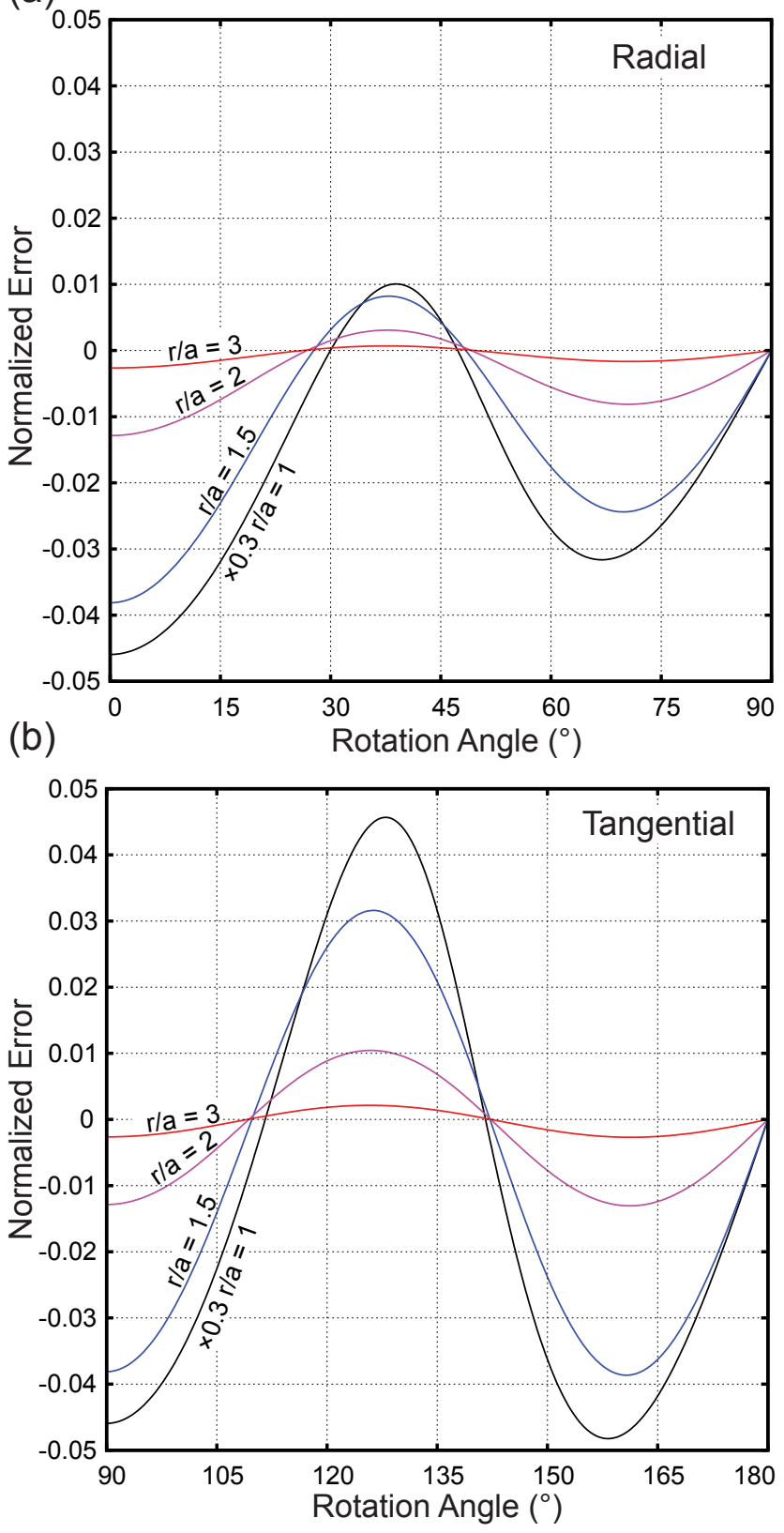